\section{OP0144 INTRAVENOUS VERSUS ORAL CYCLOPHOSPHAMIDE (CYC) FOR THE TREATMENT OF INTERSTITIAL LUNG INVOLVEMENT (ILD) AND SKIN INVOLVEMENT IN SYSTEMIC SCLEROSIS (SSC): SAFETY AND EFFICACY EVALUATION IN A LARGE MULTI-CENTRE SCLERODERMA COHORT}

C. Bruni ${ }^{1}$, D.P. Tashkin ${ }^{2}$, V. Steen ${ }^{3}$, Y. Allanore ${ }^{4}$, O. Distler ${ }^{5}$, J. Grotts ${ }^{6}$, M. MatucciCerinic $^{7}$, on behalf of EUSTAR centres, D.E. Furst ${ }^{8}$, on behalf of Scleroderma Lung Studyies I and II centers on behalf of on behalf of EUSTAR, Scleroderma Lung Study I and II centres. ${ }^{1}$ Department of Experimental and Clinical Medicine, Division of Rheumatology, University of Firenze, Firenze, Italy, ${ }^{2}$ Department of Medicine, Division of Pulmonary Medicine and Critical Care, David Geffen School of Medicine at UCLA, Los Angeles; ${ }^{3}$ Rheumatology Division, Department of Medicine, Georgetown University, Washington, USA; ${ }^{4}$ Service de Rhumatologie A, Hôpital Cochin, Université Paris Descartes, Sorbonne Paris Cité, Paris, France; ${ }^{5}$ Department of rheumatology, University Hospital Zurich, Zurich, Switzerland; ${ }^{6}$ Department of Medicine Statistics Core, University of California at Los Angeles, Los Angeles, USA; ${ }^{7}$ of Experimental and Clinical Medicine, Division of Rheumatology, University of Firenze, Firenze, Italy, ${ }^{8}$ Department of Medicine, Division of Rheumatology, University of California Los Angeles, Los Angeles, USA

Background: In the last decade, oral CYC has shown modest but significant effect on SSc-ILD and skin thickness in two large randomised controlled trials, with superiority to placebo and similar efficacy to mycophenolate. However, many centres give priority to monthly IV CYC for expected milder toxicity.

Objectives: To compare efficacy and safety of oral versus iv CYC for treating ILD and/or skin involvement in SSc.

Methods: SSc patients treated with oral or iv CYC for at least 6 months were followed for 1 year from the last administration. Data were obtained from the EUSTAR database and the Scleroderma Lung Studies I and II regarding safety [both serious (SAEs) and non-serious adverse events (AEs)] and efficacy (\%FVC, \%DLCO, mRSS) at end of treatment and after one-year follow-up were analysed [mean \pm SD or median (IQR) as appropriate].

Results: 322 patients were eligible: 149 patients received oral CYC with median daily dose 106(93-134) mg, treatment duration 365(364-366) days, while 153 patients received IV CYC median monthly dose 1000(700-1200) mg, treatment duration 335(291-374) days. Ethnicity, previous DMARD exposure, previous and concomitant steroid exposure and dosage, current/previous smoking exposure, prevalence of digital ulcers and arterial hypertension were different between the two groups (see table 1 for further details).

For efficacy: despite different baseline\%FVC and\%DLCO, adjusted changes in pulmonary measures from end of treatment (EOT) vs baseline and follow-up visit (FU) vs EOT were, respectively: \%FVC $[0(-5-5)$ vs $0(-7-7)$ and $1(-6-4)$ vs -2 $(-7-4), p=N S], \% D L C O$ [ $-4(-9-3)$ vs $-3(-9-6), 1(-6-5)$ vs $-1(-9-6), p=N S]$ and mRSS $[-3(-5-0)$ vs $-1(-5-0),-1(-4-1)$ vs $0(-3-1), p=N S]$. In a multivariate analysis, no independent variable significantly influenced\%FVC change at any visit.\%DLCO change was influenced by baseline\%DLCO and history of SScrelated cardiomyopathy at EOT assessment and by history of SSc-muscle involvement at FU visit. Baseline mRSS was the only variable having a significant impact on mRSS change.

For safety: in the oral group, there were more leukopenia (WBC $<2500 \times 10^{9} / \mathrm{mm}^{3}$ at least once $-21.6 \%$ vs $1.2 \%, p<0.001$ ), haemorrhagic cystitis [5.5\% (8 instances) vs $0 \%, p=0.011)$ at EOT visit. In contrast, there were more SAEs $(9 \%$ vs $19 \%, p=0.025)$, need for oxygen supplementation ( $5 \%$ vs $14 \%, p=0.016)$ and SSc-related cardiomyopathy onset ( $2 \%$ vs $9 \%, p=0.024)$ during follow-up in the IV group.

Abstract OP0144 - Table 1 Baseline significant differences between study population groups.

\begin{tabular}{|l|c|c|c|}
\hline & $\begin{array}{c}\text { Oral CYC } \\
(149 \mathrm{pts})\end{array}$ & $\begin{array}{c}\text { Intravenous CYC } \\
(153 \mathrm{pts})\end{array}$ & $\mathrm{p}$ \\
\hline Caucasian race, $\mathrm{n}(\%)$ & $104(69,8)$ & $146(95,4)$ & $<0,001$ \\
\hline Afro-american race, $\mathrm{n}(\%)$ & $23(15,4)$ & $0(0)$ & $<0,001$ \\
\hline Previous steroid exposure, $\mathrm{n}(\%)$ & $42(28,4)$ & $70(46,7)$ & 0,001 \\
\hline Previous DMARD exposure, $\mathrm{n}(\%)$ & $35(23,5)$ & $62(41,3)$ & 0,001 \\
\hline Steroid treatment at baseline, $\mathrm{n}(\%)$ & $51(34,6)$ & $111(72,5)$ & $<0,001$ \\
\hline CYC treatment duration (days), mean & $365(365-366)$ & $343(194-514)$ & 0,035 \\
\hline CYC daily dose $(\mathrm{mg})$, mean (IOR) & $106,6(93,1-132,3)$ & $33,3(23,3-40)$ & 0,001 \\
\hline Smoking history, $\mathrm{n}(\%)$ & $21(14,6)$ & $10(6,6)$ & 0,035 \\
\hline$\%$ FVC at baseline, mean (IOR) & $69(60-75)$ & $83(68-96)$ & $<0,001$ \\
\hline$\%$ DLco at baseline, mean (IQR) & $51(40-63)$ & $56(42-71)$ & 0,022 \\
\hline History/presence of digital ulcers, $\mathrm{n}(\%)$ & $40(26,8)$ & $64(42,1)$ & 0,008 \\
\hline History/presence of arterial & $40(26,8)$ & $25(16,3)$ & 0,035 \\
\hline CrC=cyclophosphamide, DMARD=disease modifying ant rheumatic drug, FVC=forced vital capacity, DLco= \\
\hline
\end{tabular}

Conclusions: In this hypothesis generating study, similar efficacy of one year of oral and iv CYC were seen. In contrast, a different safety profile for AE time courses and types of AEs were seen in the two groups. Although significantly higher dosage of steroids at all visits and prevalence of DMARDs use were present in the IV CYC group (as a post-treatment maintenance), these did not have an impact on either safety or efficacy. Case-control or randomised studies are warranted to extend and confirm our data.

Disclosure of Interest: None declared

DOI: 10.1136/annrheumdis-2018-eular.4674

\section{OP0145 SCLERODERMA RELATED INTERSTITIAL LUNG DISEASE AND MYCOPHENOLATE : LONG TERM OUTCOMES}

R. Janardana ${ }^{1}$, A. Irodi $^{2}$, P.P. Chebbi ${ }^{3}$, L. RV ${ }^{3}$, D. danda ${ }^{3}$, G. Mahasampath ${ }^{4}$ ${ }^{1}$ Clinical Immunology and Rheumatology, ${ }^{2}$ Radiology; ${ }^{3}$ Christian Medical College, Vellore, Vellore, India; ${ }^{4}$ Biostatistics, Christian Medical College, Vellore, Vellore. India

Background: Interstitial lung disease [ILD] is a leading cause of mortality in scleroderma. ${ }^{1}$ Scleroderma lung study-II clearly illustrates the equivalent efficacy and a better side-effect profile of mycophenolate mofetil [MMF] as compared to cyclophosphamide.

Objectives: To study the long term outcomes of mycophenolate mofetil in scleroderma related interstitial lung disease (SSc-ILD) in terms of change in forced vital capacity (FVC)

To determine the effect of MMF on longitudinal high resolution computed tomogra phy (HRCT) scores.

Methods: All patients of SSc-ILD from 2013 till date who had a baseline FVC and follow up FVC were taken for analysis. All patient received an average dose of $2 \mathrm{~g} /$ day of MMF for a median duration of 2 years and were tapered as per the standard protocol. FVC was measured using standard protocols. The FVC change was computed as percentage relative change from baseline FVC value. According to American Thoracic society recommendations, improvement is defined as an increase in FVC $\geq 10 \%$, stabilisation by change in FVC $<10 \%$ and worsening by a reduction in FVC $\geq 10 \%$

Results: We had 88 patients with a baseline and followup FVC data. Of these 66 patients had a 1 year follow up; 46 patients had a 2 year followup and 29 patients had a 3 year followup data. The absolute median (IQR) increase in the FVC value at the end of 1 year, 2 years, 3 years were $4.15(-2.3$ to 10.5$), 2.85$ (-3.4 to 7.2 and $3.8(-0.6$ to 10.4$)$ respectively. At the end of 1 year, 2 years and 3 years $89.4 \%$; $82.6 \%$ and $75.9 \%$ respectively had a stable or improved relative FVC change from baseline.

Of the 52 individuals who had a baseline as well as repeat HRCT, stable/improved scores in ground glass opacity, fibrosis and honey combing was seen in $80.8 \%$, $86.5 \%$ and $86.5 \%$ respectively. There was no difference in the extent of FVC change between those with limited vs extensive disease.

Abstract OP0145 - Table 1

\begin{tabular}{lc}
\hline Baseline Characteristics & Value \\
\hline Age, yr. mean(SD) & $33.8( \pm 11.3)$ \\
\hline Female sex, $\mathrm{n}(\%)$ & $75(85.2 \%)$ \\
Type of SSc & 5 \\
Limited & 71 \\
Diffuse & 6 \\
Sine scleroderma & $46.6( \pm 42.1)$ \\
Disease duration, months, mean (SD) & 70 \\
Antibodies & 1 \\
Scl-70 & \\
Anti centromere & $20.4( \pm 13.2)$ \\
MRSS, mean (SD) $\mathrm{n}=52$ & $61.2( \pm 17.9)$ \\
FVC\% predicted, mean (SD) & 68 \\
HRCT pattern [n=85] & 11 \\
NSIP (cellular) & 6 \\
NSIP (fibrotic) & \\
UIP & $0.33(0-1.3)$ \\
HRCT determined disease extent [median(IQR)] ; $\mathrm{n}=52$ & $1(0-1.5)$ \\
Maximum fibrosis score (0-4) & $0(0.08)$ \\
Maximum ground glass opacity (0-4) & \\
Maximum honeycombing (0-4) & $29(55.8 \%)$ \\
Maximum fibrosis score $\mathrm{n}=52$ & $23(44.2 \%)$ \\
1\%-25\% &
\end{tabular}

$26 \%-100 \%$

HRCT change over 2 years

Ground Glass opacity

Stable/lmproved

Frequency (Percentage)

$42(80.8 \%)$

Worsened

Fibrosis

$10(19.2 \%)$

Stable/Improved

$45(86.5 \%)$

$7(13.5 \%)$

Worsened

Honey combing

Stable/Improved

$45(86.5 \%)$

Worsened 